\title{
Gated time-limited polling systems
}

\author{
E. de Souza e Silva ${ }^{1}$
}

Federal University of Rio de Janeiro

Cx.P. 2324, RJ 20001-970, Brazil

H. R. Gail

IBM Thomas J. Watson Research Center

Yorktown Heights, NY 10598

\author{
R. R. Muntz $z^{1}$ \\ University of California, Los Angeles \\ Los Angeles, CA 90024
}

\begin{abstract}
Polling systems have found widespread application in the modeling of computer communication systems and protocols. As a consequence, a great deal of effort has been devoted to analyzing a variety of polling mechanisms. In particular, systems for which a limit on the length of time the server may spend while visiting a queue have recently been the subject of several studies. For example, protocols and multiplexing schemes for multimedia networks may employ a limit on the amount of time the shared channel is allocated to each type of traffic. However, such timelimited policies are difficult to analyze, and relatively few results about them have appeared in the literature. In this paper the previous methodology we developed to study the exhaustive service case with preemptive timeouts is extended and applied to the analysis of a system operating under the time-limited gated discipline and having either preemptive timeouts or nonpreemptive timeouts. Measures of interest that can be calculated include joint distributions at server arrivals, at server departures, and over all time. Additional measures of interest, such as expected waiting times and blocking probabilities for each customer class in the system, can also be computed.
\end{abstract}

\footnotetext{
${ }^{1}$ The work of E. de Souza e Silva and R. R. Muntz was partially supported by grants from National Science Foundation CCR-9215064 and from CNPq (ProTem-CC, Aux.Int., Pronex, Brazil).

Performance and Management of Complex Communication Networks T. Hasegawa, H. Takagi \& Y. Takahashi (Eds.) C 1998 IFIP. Published by Chapman \& Hall
} 


\section{INTRODUCTION}

Polling systems have been widely used to model a large variety of computer communication systems and protocols (see [1. 15]). A polling svstem consists of a set of queues that are visited by a server in a given order. The service discipline used can be one of several types, such as exhaustive, gated and customer-limited. Under the exhaustive discipline, the server serves all customers at the visited queue until it empties before moving to another queue. Under the gated discipline, the server serves only those customers that are at the queue upon its arrival, and does not serve the customers that arrive afterwards. Those customers are only served during the next visit of the server. Under the customer-limited discipline, there is a maximum number of customers that are allowed to be served during each visit to a queue. A comprehensive survey of the state of the art in the analysis of polling systems is presented in $[15,16,17]$.

Recently, several network protocols and multiplexing schemes have employed a limit on the amount of time the shared channel is allocated to a buffer queue or station. Examples of timelimited policies can be found in the ANSI/IEEE 802.5 Standard, in the IEEE 802.4 standard proposed for the token bus [18], and in the $\left(T_{1}-T_{2}\right)$ bandwidth allocation scheme for wideband packet networks proposed in [14]. Establishing a server timeout upon each visit to a queue in the polling cycle is important in order to guarantee a minimum bandwidth to different types of traffic to the system.

The $\left(T_{1}-T_{2}\right)$ multiplexing scheme was analyzed in [2], but the server timeouts were assumed to be exponentially distributed random variables, instead of the deterministic timeouts of the real system. Furthermore, it was assumed that there were no switchover times. In $[12,13]$ a single $M / G / 1$ queue with server vacations was analyzed, where the server limits the amount of time spent serving customers between vacation periods. The gated discipline was considered in [12], and it was assumed that the vacation length is a random variable which is independent of the amount of work in queue when a vacation starts. Extensions to the nongated discipline appear in [13].

In this paper we extend the methodology for analyzing time-limited systems described in [7], and show how many important details can be incorporated in the model. We consider the gated discipline in detail, and indicate how additional disciplines can be studied using the method. Both preemptive and nonpreemptive timeouts can also be taken into account. Several performance measures can be calculated using the approach, such as joint queue length distributions for arrival and departure times of the server at a specific queue, joint queue length distributions at customer arrivals and departures, limiting probabilities for the length of a particular queue in the system, the mean cycle time, and mean waiting times.

\section{PRELIMINARIES}

In this section we introduce the polling model that is studied in the paper. We also introduce the basic notation that is used and review background material that is needed. The model considered here is similar to the exhaustive service model studied in [7]. The polling system has $M$ queues, and customer arrivals at queue $i$ are governed by a Poisson process with rate $\lambda_{i}$. Customers arriving at queue $i$ bring with them exponentially distributed service demands with mean $1 / \mu_{i}$. The arrival and service processes for the $M$ queues are all independent. The buffer size at queue $i$ can be either infinite or equal to a finite value $B_{i}$. Although the service discipline may differ from queue to queue, in this paper we study the gated policy at all queues. Further, a general 
polling table may govern the movement of the server. but here we focus on the cyclic switching policy. The time required by the server to switch from queue $i$ to the next queue in sequence is assumed to be the constant value $\sigma_{i}$.

The polling system studied in this paper is time-limited. in that the server can spend at most a fixed amount of time at each queue serving customers. This const ant timeout value is equal to $T_{i}$ for queue $i$. Under the gated discipline, the server leaves and begins switching to the next queue if all customers originally in the system when the server arrived have completed service. The server also leaves when the timeout expires; in this case. the customer being serviced is preempted and returns to the queue. Due to the exponential service time assumption, the returned customer has a (revised) service requirement that is statistically identical to the other customers already waiting. In section 3.3 we consider a different policy, namely, the customer is allowed to finish service when the timeout expires (nonpreemptive timeouts). Another alternative is not to allow any customer to start service if the timeout for that queue is about ready to expire, and this system is analyzed in section 3.4. At each queue, customers may be served in any order as long as the scheduling discipline is independent of job service times.

Various performance measures are calculated for the polling system. These include joint queue length distributions at server arrivals to a particular queue, at server departures from a particular queue, at customer arrivals, and at customer departures. We also consider time average measures such as limiting marginal distributions, which in turn can be used to obtain mean waiting times of customers and, in the finite buffer case, loss probabilities.

Our interest is in the continuous-time process $\mathcal{X}=\{X(t): t \geq 0\}$, which describes the joint distribution of number in system at time $t$. A state of the process is an $M$-tuple of (nonnegative) integers $s=\left\{\left\langle q_{1}, \ldots, q_{M}\right\rangle\right\}$, where $q_{j}, j=1, \ldots, M$, represents the number of customers at queue $j$. If queue $j$ has an infinite buffer, then $q_{j}$ has values in $\{0,1, \ldots\}$ the set of nonnegative integers, while if queue $j$ has a finite buffer of size $B_{j}$, then $q_{j} \in\left\{0, \ldots, B_{j}\right\}$. Note that $X(t)$ is not a Markov process, since there are occurrences of a nonexponential nature (such as the server timeouts) in the model.

Let us look at the set of points at which the server either arrives at a queue or departs from a queue. One can obtain an embedded discrete-time Markov chain at these points by extending the state description of $\mathcal{X}$ to include an additional variable that indicates the state of the server, i.e., does the event correspond to an arrival at a queue or a departure from a queue, and which queue is it. Thus we have added a variable, say $\xi$, which can take on the possible values $\xi=1, \ldots, 2 M$. Here $\xi=2 i-1$ represents the server arriving to queue $i$, while $\xi=2 i$ represents the server departing from queue $i, i=1, \ldots, M$. Let $\mathbf{H}$ be the transition matrix for this discrete-time Markov chain embedded at points of server arrivals and server departures. Due to the cyclic nature of the polling strategy, $\mathbf{H}$ has the form

$$
\mathbf{H}=\left[\begin{array}{ccccc}
\mathbf{0} & \mathbf{D}^{(1)} & \mathbf{0} & \cdots & \mathbf{0} \\
\mathbf{0} & \mathbf{0} & \mathbf{C}^{(1)} & \cdots & \mathbf{0} \\
\vdots & \vdots & \vdots & \ddots & \vdots \\
\mathbf{0} & \mathbf{0} & \mathbf{0} & \cdots & \mathbf{D}^{(M)} \\
\mathbf{C}^{(M)} & \mathbf{0} & \mathbf{0} & \cdots & \mathbf{0}
\end{array}\right]
$$

The submatrices $\mathbf{D}^{(i)}$ and $\mathbf{C}^{(i)}$ have the following interpretation. Recall that the server alternates between serving one of the $M$ queues and switching between queues. The time during which queue $i$ is being served is called an $i$-mini-cycle, while the time during which the server is 
switching from queue $i$ to the next queue is called an $i$-switchover interval. Thus we see that the matrices $\mathbf{D}^{(i)}$ and $\mathbf{C}^{(i)}$ give the transition probabilities for the number in system over an $i$-minicycle and an $i$-switchover interval, respectively. For example, the $\left(s, s^{\prime}\right)$ entry of $\mathbf{D}^{(i)}$ gives the probability of a transition from a state $s=\left\langle q_{1}, \ldots, q_{M}, \xi\right\rangle$ at the beginning of an $i$-mini-cycle to a state $s^{\prime}=\left\langle q_{1}^{\prime}, \ldots, q_{M}^{\prime}, \xi^{\prime}\right\rangle$ at the end of an $i$-mini-cycle. Similarly, the $\left(s, s^{\prime}\right)$ entry of $\mathbf{C}^{(i)}$ gives the probability of a transition from a state $s=\left\langle q_{1}, \ldots, q_{M}, \xi\right\rangle$ at the beginning of an $i$-switchover interval to a state $s^{\prime}=\left\langle q_{1}^{\prime}, \ldots, q_{M}^{\prime}, \xi^{\prime}\right\rangle$ at the end of an $i$-switchover interval. Of course, for $\mathbf{D}^{(i)}$ we have $\xi=2 i-1$ and $\xi^{\prime}=2 i$, while for $\mathbf{C}^{(i)}$ we have $\xi=2 i$ and $\xi^{\prime}=2 i+1(\bmod 2 M)$. Thus we will disregard the variable $\xi$ in the state description in order to simplify notation.

Although $\mathbf{H}$ is cyclic, in the ergodic case a solution of the equation $\boldsymbol{\eta}=\boldsymbol{\eta} \mathbf{H}$ (up to a normalization constant) represents the long-run fraction of time of being in states of the embedded chain at server arrivals and server departures. Rewrite $\boldsymbol{\eta}$ in blocks to correspond to the matrices $\mathbf{D}^{(i)}$ and $\mathbf{C}^{(i)}$ as

$$
\eta=\left\langle\beta^{(1)}, \alpha^{(1)}, \ldots, \beta^{(M)}, \alpha^{(M)}\right\rangle .
$$

Then from the equation $\eta=\eta \mathbf{H}$ and (1), we see that

$$
\begin{array}{ll}
\boldsymbol{\beta}^{(1)}=\boldsymbol{\alpha}^{(M)} \mathbf{C}^{(M)} & \\
\boldsymbol{\beta}^{(i)}=\boldsymbol{\alpha}^{(i-1)} \mathbf{C}^{(i-1)} & i=2, \ldots, M \\
\boldsymbol{\alpha}^{(i)}=\boldsymbol{\beta}^{(i)} \mathbf{D}^{(i)} & i=1, \ldots, M .
\end{array}
$$

Now, for $i=1, \ldots, M$, let $\mathbf{H}^{(i)}$ be the transition matrix for the discrete-time embedded Markov chain for points at which the server arrives to queue $i$, i.e., for points at which an $i$-minicycle begins (and an $i-1$-switchover interval ends). Similarly, let $\mathbf{G}^{(i)}$ be the transition matrix for the discrete-time embedded Markov chain for points at which the server departs from queue $i$, i.e., for points at which an $i$-switchover interval begins (and an $i$-mini-cycle ends). Clearly we have

$$
\begin{aligned}
\mathbf{H}^{(i)} & =\mathbf{D}^{(i)} \mathbf{C}^{(i)} \ldots \mathbf{C}^{(M)} \mathbf{D}^{(1)} \ldots \mathbf{D}^{(i-1)} \mathbf{C}^{(i-1)} \\
\mathbf{G}^{(i)} & =\mathbf{C}^{(i)} \mathbf{D}^{(i+1)} \ldots \mathbf{C}^{(M)} \mathbf{D}^{(1)} \ldots \mathbf{C}^{(i-1)} \mathbf{D}^{(i)}
\end{aligned}
$$

Using (3) it is easy to show recursively that the vectors $\beta^{(i)}$ and $\alpha^{(i)}$ satisfy

$$
\begin{aligned}
& \boldsymbol{\beta}^{(i)}=\boldsymbol{\beta}^{(i)} \mathbf{H}^{(i)} \\
& \boldsymbol{\alpha}^{(i)}=\boldsymbol{\alpha}^{(i)} \mathbf{G}^{(i)} .
\end{aligned}
$$

Thus, when suitably normalized, the block entries of $\boldsymbol{\eta}$ represent the stationary probabilities for the embedded Markov chains at server arrivals to queue $i$ (i.e., $\boldsymbol{\beta}^{(i)}$ ) or server departures from queue $i$ (i.e., $\boldsymbol{\alpha}^{(i)}$ ).

In order to find these stationary vectors, one could use standard techniques to solve (5), once the transition matrices $\mathbf{H}^{(i)}$ and $\mathbf{G}^{(i)}$ are known. These matrices could be computed directly from the $\mathbf{D}^{(i)}$ and $\mathbf{C}^{(i)}$ using (4). However, it turns out that there is a more useful approach, since $\mathbf{D}^{(i)}$ and $\mathbf{C}^{(i)}$ have special structure, while $\mathbf{H}^{(i)}$ and $\mathbf{G}^{(i)}$ do not. Let $\boldsymbol{\beta}^{(i)}(n)$ and $\boldsymbol{\alpha}^{(i)}(n)$ be the values calculated at iteration $n$ using (3), e.g., $\boldsymbol{\beta}^{(1)}(n)=\boldsymbol{\alpha}^{(M)}(n-1) \mathbf{C}^{(M)}, \boldsymbol{\beta}^{(i)}(n)=\boldsymbol{\alpha}^{(i-1)}(n) \mathbf{C}^{(i-1)}$ $(i>1)$ and $\boldsymbol{\alpha}^{(i)}(n)=\boldsymbol{\beta}^{(i)}(n) \mathbf{D}^{(i)}$. To begin the iteration, the initial conditions need only specify a value for one of the $\beta^{(i)}(0)$ or $\alpha^{(i)}(0)$. In fact, we are using the power method on the $2 M$ matrices $\mathbf{H}^{(i)}$ and $\mathbf{G}^{(i)}$ simultaneously, and so this recursion converges to the quantities $\boldsymbol{\beta}^{(i)}$ and $\boldsymbol{\alpha}^{(i)}$. 
In order to employ this iteration scheme, our first task is to determine the matrices $\mathbf{D}^{(i)}$ and $\mathbf{C}^{(i)}$. Although the $\dot{\mathbf{C}}^{(i)}$ are straightforward to compute, it is more difficult to obtain the $\mathbf{D}^{(i)}$, which are the transition matrices over mini-cycles. Transient analysis is required (the length of an $i$-mini-cycle is at most $T_{i}$ ), and the method used is the uniformization technique (also called randomization or Jensen's method) $[6,10,11]$. This technique for analyzing a continuoustime Markov chain (CTMC) involves constructing a discrete-time Markov chain (DTMC) which has the same stationary distribution as one of its properties (in fact, much more is true). Let $\mathcal{U}=\{U(t): t \geq 0\}$ be a CTMC with generator $\mathbf{Q}$ and state space $\mathcal{S}$. Suppose that the entries of $\mathbf{Q}$ are uniformly bounded, i.e., there is a finite $\Lambda \geq r_{\text {s }}$ for any $s \in \mathcal{S}$, where $r_{s}$ is the rate of leaving state $s$. Consider the discrete-time Markov chain $\mathcal{V}=\left\{V_{n}: n=0,1, \ldots\right\}$, where the transition matrix of $\mathcal{V}$ is $\mathbf{P}=\mathbf{Q} / \Lambda+\mathbf{I}$. We may view $U(t)=V_{N(t)}$, where $\mathcal{N}=\{N(t): t \geq 0\}$ is a Poisson process which is independent of $\mathcal{V}$ and is of rate $\Lambda$. In terms of a probabilistic interpretation, one simply uniformizes all the rates of the CTMC to be $\Lambda$ by adding fictitious transitions from states back to themselves.

\section{EMBEDDED CHAIN DISTRIBUTIONS}

In this section we will derive expressions for the entries of the transition matrices $\mathbf{D}^{(i)}$ and $\mathbf{C}^{(i)}$ of the embedded Markov chains at server arrivals to queue $i$ and server departures from queue $i$, respectively. Our approach will be based on the methodology for time-limited polling systems with exhaustive service discipline that was studied in [7]. However, in the case considered here, the stations all have a gated discipline, which makes the analysis a bit more complicated. We first assume infinite buffer space at each queue, although the finite buffer case will be studied later in the section. Further, we first consider the case of preemptive timeouts, and then make the extension to nonpreemptive timeouts.

\subsection{Gated Service and Infinite Buffers}

Let $s=\left\langle q_{1}, \ldots, q_{M}\right\rangle \in \mathcal{S}$ and $s^{\prime}=\left\langle q_{1}^{\prime}, \ldots, q_{M}^{\prime}\right\rangle \in \mathcal{S}$ be two states of the polling system. Recall that the $i$ th entry represents the number of customers at queue $i$. The $\left(s, s^{\prime}\right)$ entry of the matrix $\mathbf{C}^{(j)}$ is denoted $c_{s, s^{\prime}}^{(j)}$, while the corresponding entry of $\mathbf{D}^{(j)}$ is $d_{s, s^{\prime}}^{(j)}$. Then $c_{s, s^{\prime}}^{(j)}$ is the probability that the number in system at the $M$ queues is $s^{\prime}$ at the end of a $j$-switchover interval given that it was $s$ when the server departed from queue $j$. Similarly $d_{s, s^{\prime}}^{(j)}$ is the probability that the system state is $s^{\prime}$ at the end of a $j$-mini-cycle given that it was $s$ when the server arrived to queue $j$.

The transition matrices $\mathbf{C}^{(j)}, j=1, \ldots, M$, do not depend on the scheduling discipline, so the results for exhaustive service also hold for the gated system. Let $k_{i}=q_{i}-q_{i}^{\prime}$, which represents the difference in the number of customers at queue $i$ between the start of the $j$-switchover interval and its end. Only arrivals occur during a switchover interval, and so clearly $c_{s, s^{\prime}}^{(j)}=0$ if $q_{i}^{\prime}<q_{i}$ for some queue $i, i=1, \ldots, M$. Otherwise, since the $j$-switchover interval has constant length $\sigma_{j}$, we have

$$
c_{s, s^{\prime}}^{(j)}=\prod_{i=1}^{M} e^{-\lambda_{i} \sigma_{j}} \frac{\left(\lambda_{i} \sigma_{j}\right)^{k_{i}}}{k_{i} !} .
$$

Note that the $c_{s, s^{\prime}}^{(j)}$ depend only on the differences $k_{i}$, and thus many of the entries of $\mathbf{C}^{(j)}$ are identical (and are located in a particular pattern). It is this type of homogeneity property that can be exploited when calculating $c_{s, s^{\prime}}^{(j)}$. 
Although the entries of $\mathbf{D}^{(j)}$ are more difficult to determine, we will see that they also satisfy a similar (but weaker) form of homogeneity. These transition probabilities describe the evolution of number in system at the various queues from the beginning of a $j$-mini-cycle to its end, while queue $j$ is being served. During the $j$-mini-cycle, only arrivals can occur at any other queue, and so the corresponding change in state depends on the length of the mini-cycle. To find the distribution of this length, $\tau_{j}$, the behavior of queue $j$ must be studied. Due to the exponential interarrival times and service demands for customers at queue $j$, the behavior of the $j$ th queue during the mini-cycle can be described by a Markov chain, say, $\mathcal{W}^{(j)}=\left\{W^{(j)}(t): t \geq 0\right\}$. Since the length of time the server spends at queue $j$ is at most $T_{j}$, a transient analysis of the Markov chain $W^{(j)}$ must be performed. In the exhaustive case, a one-dimensional chain for number in system represents this behavior, since the server leaves only if the system becomes empty or the timeout expires. However, in the gated case, only those original customers present when the server arrives are candidates for service during the mini-cycle. Thus a two-dimensional chain must be used for $\mathcal{W}^{(j)}$, which records both arrivals and departures explicitly in the state representation. That is, the state at queue $j$ must keep track of the actual number in system, but it must also specifically account for the number of original customers that are still present. These original customers determine, for example, whether the server leaves queue $j$ before the timeout is exhausted. The uniformization technique will be used for the transient analysis of $w^{(j)}$.

The state of the polling system at the end of the $j$-mini-cycle will depend on the system state when the server arrives at queue $j$ and the length of time spent serving customers. We first determine the distribution for the length of a $j$-mini-cycle; note that this length, $\tau_{j}$, only depends on the initial state of queue $j$ and not on the states of the other queues. Suppose that initially there are $q_{j}$ customers at queue $j$ when the server arrives and $q_{j}^{\prime}$ at the end of the mini-cycle. If $\tau_{j}=T_{j}$ (the timeout expires), then $q_{j}^{\prime}=0$ is not possible. However, if $\tau_{j}<T_{j}$ (the timeout does not expire), then both $q_{j}^{\prime}>0$ and $q_{j}^{\prime}=0$ may occur under gated service. By making any state for which the number of original customers drops to 0 an absorbing state, one can use uniformization over a constant length interval $T_{i}$ to find the probabilities at the end of the mini-cycle.

The continuous-time Markov chain $\mathcal{W}^{(j)}$ that describes the system behavior at queue $j$ during a $j$-minj-cycle is two-dimensional for the gated policy, with states of the form $(u, v)$, where $0 \leq v \leq u$. Here $u$ represents the actual number of customers in the served queue $j$, and $v$ is the number of original customers in that queue which are still in the system. That is, $v$ represents the number of customers that were present when the server arrived which have not yet departed, and $u$ is the sum of $v$ and the number of arrivals that have occurred. States of the form $(u, 0)$ are absorbing in $\mathcal{W}^{(j)}$, because they represent cases when the server leaves queue $j$ before the timeout expires. Since the number of original customers and the total number of customers in the system are the same at the beginning of the mini-cycle, an initial state of the chain has the form $u=v$.

Let $\nu=\left\langle\nu_{(u, v)}\right\rangle$ be an initial probability vector for the chain $\mathcal{W}^{(j)}$ at the beginning of a $j$-mini-cycle. Then $\nu_{(u, v)}=0$ if $u \neq v$. The state distribution of $\mathcal{W}^{(j)}$ at a time $t \in\left(0, T_{j}\right)$ is

$$
\mathbf{p}^{(j)}(t, \nu)=\sum_{n=0}^{\infty} e^{-\Lambda_{j: t} t} \frac{\left(\Lambda_{j} t\right)^{n}}{n !} \pi(n, \nu),
$$

where $\Lambda_{j}=\lambda_{j}+\mu_{j}$ is the uniformization rate, $\pi(0, \nu)=\nu$, and $\pi(n, \nu)=\pi(n-1, \nu) \mathbf{W}^{(j)}$, with $\mathbf{W}^{(j)}$ the transition matrix of the uniformized chain corresponding to $\mathcal{W}^{(j)}$. Once the chain 
enters an absorbing state $(u, 0)$, which occurs when the server leaves queue $j$ before the timeout expires, it remains there until the end of the uniformization interval $\left(0 . T_{j}\right)$. Thus, in particular, $\mathbf{p}^{(j)}\left(T_{j}, \nu\right)$ represents the state distribution at the end of the $j$-mini-cycle. When we assume that the length of queue $j$ is $q$ at the start of the mini-cycle, we will use the simplified notation $q$ for $\nu$. To obtain the distribution of total number of customers present when the server leaves, states with the same value of $u$ must be aggregated.

In fact, we will frequently find it numerically beneficial to partition the state space of $\mathcal{W}^{(j)}$ into absorbing and nonabsorbing states when calculating quantities of interest. For example, consider the recursion for $\pi(n, \nu)$ from equation (7). Write the transition matrix $\mathbf{W}^{(j)}$ of the uniformized Markov chain $\mathcal{W}^{(j)}$ in blocks with respect to the absorbing states as

$$
\mathbf{W}^{(j)}=\left[\begin{array}{cc}
\mathbf{I}_{\mathrm{abs}} & \mathbf{0} \\
\mathbf{u} & \mathbf{U}^{(j)}
\end{array}\right] \text {. }
$$

Also, write $\pi(n, \nu)=\langle\psi(n, \nu), \theta(n, \nu)\rangle$. The vectors $\psi$ and $\theta$ are associated with the absorbing and nonabsorbing states, respectively. Then we calculate $\theta(n, \nu)=\theta(n-1, \nu) U^{(j)}, \phi(n, \nu)=$ $\theta(n-1, \nu) u$, and $\psi(n, \nu)=\psi(n-1, \nu)+\phi(n, \nu)$. Here, the vector $\phi(n, \nu)$ represents the probability of entering an absorbing state at step $n$ of the recursion for $\pi$. Note that $\phi(n, \nu)=$ $\psi(n, \nu)-\psi(n-1, \nu)$ may be computed recursively in a numerically stable manner without subtractions as shown above.

We now find the distribution of $\tau_{j}$, the length of a $j$-mini-cycle. First, the probability that $\tau_{j}=0$ is the probability that the server arrives to find queue $j$ empty, and so

$$
P\left[\tau_{j}=0 \mid \nu\right]=\nu_{(0,0)} .
$$

Further, the probability that $\tau_{j}=T_{j}$ is the probability that at least one of the original customers does not complete service before the timeout expires, i.e., the Markov chain $\mathcal{W}^{(j)}$ does not enter an absorbing state before time $T_{j}$. Thus

$$
P\left[\tau_{j}=T_{j} \mid \nu\right]=\sum_{n=0}^{\infty} e^{-\Lambda, T_{j}} \frac{\left(\Lambda_{j} T_{j}\right)^{n}}{n !}\|\theta(n, \nu)\| .
$$

We have used the notation $\|v\|$ for the $l_{1}$ norm of the vector $v$, i.e., for the sum of its entries. Now consider the final case, for which the server arrives to a nonempty system and serves all original customers to completion before the timeout period ends. Let

$$
F(t, \nu)=P\left[\tau_{j} \leq t \mid \nu\right], \quad 0<t<T_{j} .
$$

In terms of the Markov chain $\mathcal{W}^{(j)}, F(t, \nu)$ is the probability of being in one of the absorbing states $(u, 0)$ at time $t$. For $0<t<T_{j}$, we have

$$
F(t, \nu)=\sum_{n=0}^{\infty} e^{-\Lambda_{j} t} \frac{\left(\Lambda_{j} t\right)^{n}}{n !}\|\psi(n, \nu)\| .
$$

Here $\|\psi(n, \nu)\|$ is the probability of having no original customers left in the system at step $n$ of the uniformized chain corresponding to $W^{(j)}$. Differentiating this expression gives the density $F^{\prime}(t, \nu)$, for $0<t<T_{j}$, as

$$
F^{\prime}(t, \nu)=\sum_{n=1}^{\infty} e^{-\Lambda_{j} t} \frac{\left(\Lambda_{j} t\right)^{n-1}}{(n-1) !} \Lambda_{j}\|\phi(n, \nu)\| .
$$


For any absorbing state $(u, 0), \phi_{(u, 0)}(n, \nu)=\psi_{(u, 0)}(n, \nu)-\psi_{(u, 0)}(n-1, \nu)$ is simply the probability of being absorbed in state $(u, 0)$ at exactly the $n$th step of the uniformized Markov chain, i.e., of having $u$ customers in the system with the last original customer departing at step $n$.

Let $s=\left\langle q_{1}, \ldots, q_{M}\right\rangle$ and $s^{\prime}=\left\langle q_{1}^{\prime} \ldots, q_{M}^{\prime}\right\rangle$ be two states of the polling system, where recall that the $j$ th entry represents the number in system at queue $j$. Also let $k_{i}=q_{i}-q_{i}^{\prime}$ for $i=$ $1, \ldots, M$. To determine $d_{s, s^{\prime}}^{(j)}$, the distribution $F(t, \nu)$ of $\tau_{j}$ will be used. In the gated case, there may be customers present at queue $j$ at the end of a $j$-mini-cycle even when the server leaves before the timeout period expires, i.e., the case $q_{j}^{\prime}>0$ and $0<\tau_{j}<T_{j}$ may occur unlike the exhaustive system. However, the case $q_{j}^{\prime}=0$ and $\tau_{j}=T_{j}$ cannot occur in either system. Let $d_{s, s^{\prime}, 0}^{(j)}$ be the transition probabilities corresponding to the case for which the timeout does not expire (i.e., $0<\tau_{j}<T_{j}$ ). Similarly, let $d_{s, s^{\prime}, 1}^{(j)}$ be the transition probabilities corresponding to the case for which the timeout does expire (i.e., $\tau_{j}=T_{j}$ ). Then we have

$$
d_{s, s^{\prime}}^{(j)}=d_{s, s^{\prime}, 0}^{(j)}+d_{s, s^{\prime}, 1}^{(j)} \text {. }
$$

Of course, $d_{s, s^{\prime}}^{(j)}=0$ when $q_{i}^{\prime}<q_{i}$ for some $i \neq j$. This holds since $k_{i}=q_{i}^{\prime}-q_{i} \geq 0$ is the number of arrivals at queue $i(i \neq j)$ during the $j$-mini-cycle.

For $\tau_{j}=T_{j}$, if $q_{j}^{\prime}=0$, then $d_{s, s^{\prime}, 0}^{(j)}=0$. But if $q_{j}^{\prime}>0$, the uniformized chain $\mathcal{W}^{(j)}$ must be in one of the nonabsorbing states $\left(q_{j}^{\prime}, v\right), v \geq 1$, at time $T_{j}$, and we obtain

$$
\begin{aligned}
d_{s, s^{\prime}, 1}^{(j)}= & \sum_{n=\kappa_{j}}^{\infty} \epsilon^{-\gamma_{j} T} \frac{\left(\gamma_{j} T_{j}\right)^{n}}{n !} \prod_{i \neq j}\left(\frac{\lambda_{i}}{\gamma_{j}}\right)^{k_{i}}\left(\frac{\Lambda_{j}}{\gamma_{j}}\right)^{n-\kappa_{j}} \\
& \left.\times \frac{n !}{\left(n-\kappa_{j}\right) ! \prod_{i \neq j} k_{i} !} \sum_{v=1}^{q_{j}^{\prime}} \pi_{\left(q_{j}^{\prime}, v\right)}\right)\left(n-\kappa_{j},\left(q_{j}, q_{j}\right)\right) .
\end{aligned}
$$

Here $\kappa_{j}=\sum_{i \neq j} k_{i}, \Lambda_{j}=\lambda_{j}+\mu_{j}$, and $\gamma_{j}=\sum_{i \neq j} \lambda_{i}+\Lambda_{j}=\sum_{i=1}^{M} \lambda_{i}+\mu_{j}$. To justify this result, consider the superposition of $M$ independent Poisson processes. The $i$ th process, for $i \neq j$, has rate $\lambda_{i}$ and represents arrivals to queue $i$. The $j$ th process has rate $\Lambda_{j}$ and represents transitions of the two-dimensional uniformized chain for queue $j$ corresponding to $\mathcal{W}^{(j)}$. The $n$th term of the series on the righthand side of (12) gives the probability that $n$ transitions in the aggregate process occurred during the $j$-mini-cycle of length $T_{j}$. Of these $n$ events, $k_{i}$ arrivals occurred at queue $i, i \neq j$, while $n-\kappa_{j}$ transitions of the chain $\mathcal{W}^{(j)}$ occurred, and this uniformized chain was in state $q_{j}^{\prime}$ at time $T_{j}$ (the end of the timeout period).

For the case $0<\tau_{j}<T_{j}$, both $q_{j}^{\prime}>0$ and $q_{j}^{\prime}=0$ are possible. The state of the chain $\mathcal{W}^{(j)}$ at time $T_{j}$ is the absorbing state $\left(q_{j}^{\prime}, 0\right)$. To find $d_{s, s^{\prime}, 0}^{(j)}$, we condition on $\tau_{j}$ and obtain

$$
\begin{aligned}
d_{s, s^{\prime}, 0}^{(j)}= & \sum_{n=\kappa_{j}+1}^{\infty} e^{-\gamma j T_{j}} \frac{\left(\gamma_{j} T_{j}\right)^{n}}{n !} \sum_{m=\kappa_{j}+1}^{n} \prod_{i \neq j}\left(\frac{\lambda_{i}}{\gamma_{j}}\right)^{k_{i}}\left(\frac{\Lambda_{j}}{\gamma_{j}}\right)^{m-\kappa_{j}} \\
& \times \frac{(m-1) !}{\left(m-1-\kappa_{j}\right) ! \prod_{i \neq j} k_{i} !} \phi_{\left(q_{j}^{\prime}, 0\right)}\left(m-\kappa_{j},\left(q_{j}, q_{j}\right)\right) .
\end{aligned}
$$

This equation can be justified in the following way. As before, consider the superposition of $M$ independent Poisson processes of rates $\lambda_{i}, i \neq j$, and $\Lambda_{j}$. Then, in (13) above, index $n$ gives the number of transitions of this aggregate process during an interval of length $T_{j}$, while $m$ represents the transition at which the process entered an absorbing state (i.e., the transition at 
which the server finished service and departed from queue $j$ ). For $i \neq j, k_{i}$ arrivals to queue $i$ must have occurred, while $m-\kappa_{j}$ transitions of the uniformized chain for queue $j$ occurred until the server left (these,consisted of arrivals, departures and fictitious self-transitions). The final one of these transitions was of type $j$ and corresponded to queue $j$ having no more original customers to serve. If $m<n$, additional transitions of the superposed process occurred after the server departed from queue $j$, but still during the interval of length $T_{j}$. However, these were simply transitions from one of the absorbing states of $\mathcal{W}^{(j)}$ back to itself, and thus did not affect the final state of the polling system.

The expressions for $c_{s, s^{\prime}}^{(j)}$ and $d_{s, s^{\prime}}^{(j)}$ derived above involve the Poisson distribution, which has a general term of the form $e^{-\lambda T}(\lambda T)^{n} / n$ !, where $\lambda$ and $T$ are given parameters. Efficient and numerically stable-schemes for calculating this distribution have been developed (see $[5,8])$. The infinite series that appear in the expressions for $d_{s, s^{\prime}, 0}^{(j)}$ and $d_{s, s^{\prime}, 1}^{(j)}$ must be truncated when performing numerical calculations. However, the error resulting from such a truncation can be bounded ahead of time (see [9]).

\subsection{Gated Service and Finite Buffers}

When the buffer sizes at the $M$ queues are finite, the above arguments must be modified to obtain the elements of the transition matrices $\mathbf{C}^{(j)}$ and $\mathbf{D}^{(j)}$. Let $B_{i}<+\infty$ be the buffer size at queue $i$. As before, we need only consider pairs of states for which $q_{i}^{\prime} \geq q_{i}$ for all $i$. To find the $\mathbf{C}^{(j)}, j=1, \ldots, M$, note that the previous result for the infinite buffer system can be used for those queues $i$ for which the entry $q_{i}^{\prime}<B_{i}$. But if $q_{i}^{\prime}=B_{i}$, we must take into account an infinite number of possibilities for the number of arrivals at queue $i$, i.e., as long as there are at least $B_{i}-q_{i}$ arrivals, the final state at queue $i$ will be $B_{i}$. We partition the set of queues based on this observation, namely, define $I_{1}=\left\{i: q_{i}^{\prime}<B_{i}\right\}$ and $I_{2}=\left\{i: q_{i}^{\prime}=B_{i}\right\}$. Also, for $i=1, \ldots, M$, let $k_{i}^{*}=q_{i}^{\prime}-q_{i}$. From the above discussion, we obtain

$$
c_{s, s^{\prime}}^{(j)}=\left[\prod_{i \in I_{1}} e^{-\lambda_{i} \sigma_{j}} \frac{\left(\lambda_{i} \sigma_{j}\right)^{k_{i}^{*}}}{k_{i}^{* !} !}\right]\left[\prod_{i \in I_{2}} \sum_{k_{i}=k_{i}^{*}}^{\infty} e^{-\lambda_{i} \sigma_{j}} \frac{\left(\lambda_{i} \sigma_{j}\right)^{k_{i}}}{k_{i} !}\right] .
$$

For $i \in I_{2}, k_{i} \geq q_{i}^{\prime}-q_{i}=B_{i}-q_{i}$ represents the number of arrivals at queue $i$ during the switchover interval. Again note that the entries of the $\mathbf{C}^{(j)}$ satisfy a homogeneity property.

We next turn our attention to finding the $\mathbf{D}^{(j)}, j=1, \ldots, M$. The procedure that was followed in the infinite buffer case involved first determining the distribution of $\tau_{j}$, the length of a $j$-mini-cycle, and then using it to find the entries $d_{s, s^{\prime}}^{(j)}$. When queue $j$ has a finite buffer size of $B_{j}$, the state space of the two-dimensional chain $\mathcal{W}^{(j)}$ is truncated so that the coordinates are at most $B_{j}$ (note that state $\left(B_{j}, 0\right)$ is not reachable). Equations (9) and (10) again give the distribution of $\tau_{j}$, but the vectors $\pi(n)$ are now obtained from the transition matrix $\mathbf{W}^{(j)}$ of the uniformized chain corresponding to the truncated two-dimensional chain.

Once the distribution of $\tau_{j}$ has been determined, it remains to find analogues of equations (12) and (13) for $d_{s, s^{\prime}}^{(j)}$. We only consider the case $q_{i}^{\prime} \geq q_{i}, i \neq j$, since otherwise $d_{s, s^{\prime}}^{(j)}=0$. Recall that for infinite buffers the transition probabilities $d_{s, s^{\prime}}^{(j)}$ are a function of the length of queue $i$ $(i \neq j)$ only through the difference $q_{i}^{\prime}-q_{i}$. However, this is not true in the finite buffer case, since this difference does not necessarily represent the number of arrivals to queue $i$ during the $j$-mini-cycle. For example, if a state $s^{\prime}$ has its $i$ th entry at the buffer limit for some $i \neq j$, i.e., 
$q_{i}^{\prime}=B_{i}$, then a transition from $s$ to $s^{\prime}$ represents the infinite set of cases for which there are at least $B_{i}-q_{i}$ arrivals at queue $i$.

If $q_{i}^{\prime}<B_{i}$ for all $i \neq j$, then the equations for the infinite buffer case may be used, but if $q_{i}^{\prime}=B_{i}$ for some $i \neq j$, then we need to sum infinitely many of those equations to account for all relevant arrival patterns. For states $s$ and $s^{\prime}$, let $\dot{k}_{i}^{*}=q_{i}^{\prime}-q_{i}$ be the difference in the state of queue $i$ at the beginning and the end of the mini-cycle, and let $\kappa_{j}^{*}=\sum_{i \neq j} k_{i}^{*}$. Also let $k_{i}$ represent the number of arrivals to queue $i, i \neq j$, and let $a=\sum_{i \neq j} k_{i}$ represent the total number of arrivals to the nonserved queues. Define the set of vectors

$$
\mathbf{K}_{s, \prime^{\prime}}^{(j)}(a)=\left\{\left\langle k_{1}, \ldots, k_{j-1}, k_{j+1}, \ldots, k_{M}\right): \sum_{i \neq j} k_{i}=a . k_{i}=k_{i}^{*} \text { if } q_{i}^{\prime}<B_{i}, k_{i} \geq k_{i}^{*} \text { if } q_{i}^{\prime}=B_{i}\right\},
$$

which corresponds to all possible arrivals that give a state change from $s$ to $s^{\prime}$.

For $\tau_{j}=T_{j}$, we must have $q_{j}^{\prime}>0$, and the final state of $\mathcal{W}^{(j)}$ must have the form $\left(q_{j}^{\prime}, v\right)$, $1 \leq v \leq q_{j}^{\prime}$. From (13), we have

$$
\begin{aligned}
d_{s, s^{\prime}, 1}^{(j)}= & \sum_{n=\kappa_{j}^{*}}^{\infty} e^{-\gamma_{j} T_{j}} \frac{\left(\gamma_{j} T_{j}\right)^{n}}{n !} \sum_{a=\kappa_{j}^{*}}^{n} \sum_{k \in K_{,,,^{\prime}}^{(j)}(a)} \prod_{i \neq j}\left(\frac{\lambda_{i}}{\gamma_{j}}\right)^{k_{i}}\left(\frac{\Lambda_{j}}{\gamma_{j}}\right)^{n-a} \\
& \times \frac{n !}{(n-a) ! \prod_{i \neq j} k_{i} !} \sum_{v=1}^{q_{j}^{\prime}} \pi_{\left(q_{j}^{\prime}, v\right)}\left(n-a,\left(q_{j}, q_{j}\right)\right) .
\end{aligned}
$$

Here $n$ represents the number of transitions of the superposition of the uniformized chain for queue $j$ and the Poisson arrival processes to queues $i \neq j$. Equation (15) is obtained by conditioning on the number of events $n$ during an interval of length $T_{j}$ and grouping the conditional probabilities according to the total number of arrivals $a$ to the nonserved queues.

For $0<\tau_{j}<T_{j}$, then $q_{j}^{\prime} \geq 0$ is possible, and the final state of $\mathcal{W}^{(j)}$ has the form $\left(q_{j}^{\prime}, 0\right)$. From (12), we have

$$
\begin{aligned}
& d_{s, \varepsilon^{\prime}, 0}^{(j)}=\sum_{n=\kappa_{j}^{*}+1}^{\infty} e^{-\gamma_{j} T_{j}} \frac{\left(\gamma_{j} T_{j}\right)^{n}}{n !} \sum_{a=\kappa_{j}^{*}}^{n-1} \sum_{\mathbf{k} \in \mathbf{K}_{a, \theta^{\prime}}^{(j)}(a)} \sum_{m=a+1}^{n} \prod_{i \neq j}\left(\frac{\lambda_{i}}{\gamma_{j}}\right)^{k_{i}}\left(\frac{\Lambda_{j}}{\gamma_{j}}\right)^{m-a} \\
& \times \frac{(m-1) !}{(m-1-a) ! \prod_{i \neq j} k_{i} !} \phi_{\left(q_{j}^{\prime}, 0\right)}\left(m-a,\left(q_{j}, q_{j}\right)\right) .
\end{aligned}
$$

By interchanging the order of summation, we may also write these equations in the form (for $\left.\tau_{j}=T_{j}\right)$

$$
\begin{aligned}
d_{s, s^{\prime}, 1}^{(j)}= & \sum_{a=\kappa_{j}^{\prime}}^{\infty} \sum_{n=a}^{\infty} e^{-\gamma_{j} T_{j}} \frac{\left(\gamma_{j} T_{j}\right)^{n}}{n !} \sum_{k \in K_{s_{,}^{\prime},}^{(j)}(a)} \prod_{i \neq j}\left(\frac{\lambda_{i}}{\gamma_{j}}\right)^{k_{i}}\left(\frac{\Lambda_{j}}{\gamma_{j}}\right)^{n-a} \\
& \times \frac{n !}{(n-a) ! \prod_{i \neq j} k_{i} !} \sum_{v=1}^{q_{j}^{\prime}} \pi_{\left(q_{j}^{\prime}, v\right)}\left(n-a,\left(q_{j}, q_{j}\right)\right),
\end{aligned}
$$

and (for $0<\tau_{j}<T_{j}$ )

$$
\begin{aligned}
d_{s, s^{\prime}, 0}^{(j)}= & \sum_{a=\kappa_{j}^{*}}^{\infty} \sum_{n=a+1}^{\infty} e^{-\gamma_{j} T_{j}} \frac{\left(\gamma_{j} T_{j}\right)^{n}}{n !} \sum_{k \in K_{,,,^{\prime}}^{(j)}(a)} \sum_{m=a+1}^{n} \prod_{i \neq j}\left(\frac{\lambda_{i}}{\gamma_{j}}\right)^{k_{i}}\left(\frac{\Lambda_{j}}{\gamma_{j}}\right)^{m-a} \\
& \times \frac{(m-1) !}{(m-1-a) ! \prod_{i \neq j} k_{i} !} \phi_{\left(q_{j}^{\prime}, 0\right)}\left(m-a,\left(q_{j}, q_{j}\right)\right) .
\end{aligned}
$$




\subsection{Nonpreemptive Timeouts}

For the case of preemptive timeouts, recall that the customer in service at the end of a timeout is returned to the set of waiting customers by assumption. If the customer is not returned when the timeout expires but instead is allowed to complete service (thus extending the visit of the server for more than $T_{j}$ time units), then the following modification is required. Uniformization is used on the chain $\mathcal{W}^{(j)}$ for an interval of length $T_{j}$. If the queue empties before the server timeout $T_{j}$ expires, then one proceeds as before. However, if the server timeout does expire, then arrivals to all queues will continue while the customer in service is permitted to finish, and the number of additional arrivals is independent of the system state at time $T_{j}$. If $q_{j}^{\prime}$ is the state of the served queue $j$ at $T_{j}$, then the state at the end of the $j$-mini-cycle will be $q_{j}^{\prime \prime}$ if there are $q_{j}^{\prime \prime}-q_{j}^{\prime}+1$ customers that arrive to the $j$ th queue after $T_{j}$. Also, for the nonserved queues $i \neq j$, if $q_{i}^{\prime}$ is the state of the queue at $T_{j}$, the state at the end of the $j$-mini-cycle is $q_{i}^{\prime \prime}$ if there are $q_{i}^{\prime \prime}-q_{i}^{\prime}$ arrivals to queue $i$ during the overrun.

Since exponential service time distributions have been assumed, the residual service time (overrun length) is exponential with mean $1 / \mu_{j}$. Furthermore, the random variables $\zeta_{i}, i=$ $1, \ldots, M$, which represent the additional arrivals at queue $i$, are independent given the length of the overrun. These properties make it easy to calculate the distribution of the random vector $\left\langle\zeta_{1}, \ldots, \zeta_{M}\right\rangle$. In fact, one sees that

$$
P\left[\zeta_{1}=v_{1}, \ldots, \zeta_{M}=v_{M}\right]=\left(\frac{\mu_{j}}{\gamma_{j}}\right) \prod_{i=1}^{M}\left(\frac{\lambda_{i}}{\gamma_{j}}\right)^{v_{i}} \frac{\left(v_{1}+\cdots+v_{M}\right) !}{v_{1} ! \cdots v_{M} !}
$$

where recall that $\gamma_{j}=\sum_{i=1}^{M} \lambda_{i}+\mu_{j}$. Let $\mathbf{E}^{(j)}=\left[e_{s^{\prime}, s^{\prime \prime}}^{(j)}\right]$ be the matrix which gives the transition probabilities from state $s^{\prime}$ at time $T_{j}$ to state $s^{\prime \prime}$ at the end of the $j$-mini-cycle. Then $e_{s^{\prime}, s^{\prime \prime}}^{(j)}=0$ unless $q_{i}^{\prime \prime}-q_{i}^{\prime} \geq 0$ for $i \neq j$ and $q_{j}^{\prime \prime}-q_{j}^{\prime}+1 \geq 0$. In this case

$$
e_{s^{\prime}, s^{\prime \prime}}^{(j)}=P\left[\zeta_{1}=q_{1}^{\prime \prime}-q_{1}^{\prime}, \ldots, \zeta_{j}=q_{j}^{\prime \prime}-q_{j}^{\prime}+1, \ldots, \zeta_{M}=q_{M}^{\prime \prime}-q_{M}^{\prime}\right] .
$$

The transition probabilities for the $j$-mini-cycle may now be found using the above information. Let $f_{s, s^{\prime \prime}}^{(j)}$ be the transition probabilities from the beginning of the mini-cycle to its end (including overruns). Consider the infinite buffer case, when service at queue $j$ is given in a gated fashion with nonpreemptive timeouts. If the server left before the timeout expired $\left(0<\tau_{j}<T_{j}\right)$, then no overrun occurred and necessarily $q_{j}^{\prime \prime}=q_{j}^{\prime}$. If the timeout did expire, then there must have been $q_{j}^{\prime \prime}-q_{j}^{\prime}+1$ arrivals during the overrun (recall that $q_{j}^{\prime}>0$ is required). In either case, both $q_{j}^{\prime \prime}>0$ and $q_{j}^{\prime \prime}=0$ are possible. In fact, we have

$$
f_{s, s^{\prime \prime}}^{(j)}=d_{s, s^{\prime \prime}, 0}^{(j)}+\sum_{s^{\prime}: 0<q_{j}^{\prime} \leq q_{j}^{\prime \prime}+1} d_{s, s^{\prime}, 1}^{(j)} e_{s^{\prime}, s^{\prime \prime}}^{(j)},
$$

where $d_{s, s^{\prime \prime}, 0}^{(j)}$ and $d_{s, s^{\prime \prime}, 1}^{(j)}$ are calculated using the gated preemptive equations (12) and (13).

\subsection{Reducing the Timeout Interval}

If a departure occurs from the served queue $j$ when the timeout interval has almost expired, then the next customer to be served (if any) will probably not complete service before $T_{j}$. Thus, either an overrun will occur for the nonpreemptive policy, or the customer will be returned to the waiting line for the preemptive policy. Both cases may adversely affect the system performance, 
since the probable overrun may increase the delay of customers of other queues in the system in the nonpreemptive case (as will be seen in the examples we give), or some transmission capacity will be wasted in the preemptive case. Therefore, it may be advantageous for the server to immediately begin switching to the next queue near the end of the timeout period, instead of accepting an additional customer into service. These considerations give rise to the following extension of the basic time-limited polling system. We assume that there is a constant $\omega_{j}$ for queue $j, j=1, \ldots, M$, such that if the timeout interval has exceeded $T_{j}-\omega_{j}$ but has not yet expired by reaching $T_{j}$, then no new customer will be allowed to enter service. Thus, if any customer finishes in the interval $\left(T_{j}-\omega_{j}, T_{j}\right)$, the server leaves queue $j$ and begins to switch to the next queue.

It is interesting to observe that the extension above provides no additional generality in the nonpreemptive timeout case. To see this, consider a $j$-mini-cycle and note that any customer in service at time $T_{j}-\omega_{j}$ will be served to completion in this new system, and no additional customers will be accepted into service. We may thus consider the remaining service time of this customer after $T_{j}-\omega_{j}$ simply as an "overrun," during which arrivals to all queues continue to occur. Thus the behavior of the new system is seen to be equivalent to one with nonpreemptive timeouts and with a timeout period of length $T_{j}-\omega_{j}$.

The preemptive timeout case, however, leads to a more general system than those we considered previously. Suppose there is a customer in service at time $T_{j}-\omega_{j}$ in the served queue $j$. Then either the customer departs before time $T_{j}$ and the server begins switching to the next queue, or the customer does not finish service before the timeout expires and is preempted back to the waiting line at time $T_{j}$. Thus the system is similar to one with nonpreemptive timeouts and an "overrun," the length of which is independent of the state at $T_{j}-\omega_{j}$ and is given by an exponential random variable of mean $\mu_{j}$ truncated at $\omega_{j}$. That is, given that the served queue $j$ is not empty at $T_{j}-\omega_{j}$ during a $j$-mini-cycle, the "overrun" length $\chi_{j}$ has distribution $P\left[\chi_{j} \leq t\right]=1-e^{-\mu_{j} t}$ for $t<\omega_{j}$, and $P\left[\chi_{j} \leq \omega_{j}\right]=1$. However, this system differs from the nonpreemptive timeout case, since the customer in service at $T_{j}-\omega_{j}$ only leaves the system if $\chi_{j}<\omega_{j}$ and is returned to the waiting line if $\chi_{j}=\omega_{j}$.

\section{TIME AVERAGE DISTRIBUTIONS}

In the previous section, we derived expressions for the entries of the matrices $\mathbf{D}^{(j)}$ and $\mathbf{C}^{(j)}$. The stationary probability vectors $\beta^{(j)}$ and $\boldsymbol{\alpha}^{(j)}$ of the embedded Markov chains at times when the server arrives to queue $j$ and when the server leaves from queue $j$, respectively, can be computed from these matrices recursively. In this section we show how $\beta^{(j)}$ and $\boldsymbol{\alpha}^{(j)}$ can be used to calculate time average distributions. We consider the infinite buffer case, since the procedure for the finite buffer case is similar. We also assume preemptive timeouts, although it is possible to extend the analysis to the nonpreemptive timeout case, as was done for the embedded chain distributions.

Let $s=\left\langle q_{1}, \ldots, q_{M}\right\rangle$ be a state of the process $X(t)$, and suppose we want to find the limiting probability that the system is in state $s$, i.e., $\lim _{t \rightarrow \infty} P[X(t)=s]$. More generally, let $\mathcal{L} \subset \mathcal{S}$ be a subset of states, and we seek $P_{\mathcal{L}}=\lim _{t \rightarrow \infty} P[X(t) \in \mathcal{L}]$ We assume that the polling system is stable in the sense that the $P_{\mathcal{L}}$ exist. Although the process $X(t)$ is not Markovian, it is regenerative with regeneration points, for example, at the times when the server visits a particular queue $i$ and there are no customers at any of the $M$ queues. Thus $P_{\mathcal{L}}$ is the ratio of the expected time spent in the set of states $\mathcal{L}$ during a regeneration cycle (the time between two 
regeneration points) to the expected length of a cycle.

However, an expression for $P_{\mathcal{L}}$ can also be given in terms of the $2 M$ stationary vectors $\beta^{(j)}$ and $\boldsymbol{\alpha}^{(j)}, j=1, \ldots, M$, and expectations of certain random variables associated with mini-cycles and switchover intervals. Define $U_{s}^{(j)}$ to be the time spent in $\mathcal{L}$ during a $j$-mini-cycle given that $s$ was the state at the start of the mini-cycle, and define $V_{s}^{(j)}$ to be the time spent in $\mathcal{L}$ during a $j$-switchover interval given that the state at the start of the interval was $s$. Similarly, define $B_{s}^{(j)}$ to be the length of a $j$-mini-cycle given that $s$ was the state at the start of the mini-cycle, and define $A_{s}^{(j)}$ to be the length of a $j$-switchover interval given that the state at the start of the interval was $s$. It is shown in [7] using results from Markov chains with rewards that

$$
P_{\mathcal{L}}=\frac{\sum_{j=1}^{M} \sum_{s \in \mathcal{S}} E\left[l_{s}^{(j)}\right] \beta_{s}^{(j)}+\sum_{j=1}^{M} \sum_{s \in \mathcal{S}} E\left[V_{s}^{(j)}\right] \alpha_{s}^{(j)}}{\sum_{j=1}^{M} \sum_{s \in \mathcal{S}} E\left[B_{s}^{(j)}\right] \beta_{s}^{(j)}+\sum_{j=1}^{M} \sum_{s \in \mathcal{S}} E\left[A_{s}^{(j)}\right] \alpha_{s}^{(j)}}
$$

This equation has the following intuitive explanation. Consider the expected time that $X(t)$ remains in $\mathcal{L}$ during a $j$-mini-cycle or a $j$-switchover interval given the state at the beginning of the particular time period of interest, and weight these quantities by the entries of the stationary vector of the corresponding embedded Markov chain. Then sum the resulting $2 M$ expected values and divide by the expected length of the polling cycle (the amount of time required by the server to complete one cycle of the queues). This gives the long-run fraction of time that the process is in the set of states $\mathcal{L}$.

\subsection{Gated Service and Infinite Buffers}

We will now apply (20) to find the limiting distribution of number of customers at a particular queue, say queue $i$. In this case the set $\mathcal{L}$ consists of those states for which the $i$ th entry has the same value, say $q$. Assuming that the stationary vectors $\beta^{(j)}$ and $\alpha^{(j)}$ have been calculated as in the previous section, we need to find expressions for $E\left[U_{s}^{(j)}\right], E\left[V_{s}^{(j)}\right], E\left[B_{s}^{(j)}\right]$, and $E\left[A_{s}^{(j)}\right]$ corresponding to this marginal distribution.

The length of a $j$-switchover interval is constant, and so $E\left[A_{s}^{(j)}\right]=\sigma_{j}$ for any state $s$. Thus we have

$$
\sum_{j=1}^{M} \sum_{s \in \mathcal{S}} E\left[A_{s}^{(j)}\right] \alpha_{s}^{(j)}=\sum_{j=1}^{M} \sigma_{j}
$$

which is the total amount of time during a polling cycle that the server spends switching from queue to queue. Of course, this is the same as the result obtained in [7] for the exhaustive case, since the scheduling discipline does not affect the switchover times.

The expected length of a $j$-mini-cycle differs in the gated and exhaustive cases. However, as in the exhaustive case, the quantity $E\left[B_{s}^{(j)}\right]$ depends only on the $j$ th entry of the initial state $s$, since the number of customers present at any queue other than $j$ does not influence the length of the $j$-mini-cycle. Thus all states $s=\left\langle q_{1}, \ldots, q_{M}\right\rangle$ with the same $j$ th entry $q$ yield the same value of $E\left[B_{s}^{(j)}\right]$. For $q=0,1, \ldots$, partition the state space into the sets $\mathcal{S}_{q}^{(j)}=\left\{\left\langle q_{1}, \ldots, q_{M}\right\rangle \in \mathcal{S}: q_{j}=q\right\}$. Note that for any $s \in \mathcal{S}_{q}^{(j)}$, we have $E\left[B_{s}^{(j)}\right]=E\left[\tau_{j} \mid q\right]$. Therefore,

$$
\sum_{s \in \mathcal{S}} E\left[B_{s}^{(j)}\right] \beta_{s}^{(j)}=\sum_{q=0}^{\infty} \sum_{s \in \mathcal{S}_{q}^{(j)}} E\left[\tau_{j} \mid q\right] \beta_{s}^{(j)}=\sum_{q=0}^{\infty} E\left[\tau_{j} \mid q\right] b_{q}^{(j)}=E\left[\tau_{j} \mid \mathbf{b}^{(j)}\right]
$$


Here we have defined the vector $\mathbf{b}^{(j)}=\left\langle b_{0}^{(j)}, b_{1}^{(j)}, \ldots\right\rangle$, where (for $q=0.1 \ldots$ )

$$
b_{q}^{(j)}=\sum_{s \in \mathcal{S}_{q}^{(j)}} \beta_{s}^{(j)} .
$$

Note that $\mathbf{b}^{(j)}$ is simply the stationary distribution of the number of customers at queue $j$ at times when the server arrives to queue $j$.

Given the initial distribution $\mathbf{b}^{(j)}$, the distribution of $\tau_{j}$, the length of a $j$-mini-cycle, is $F\left(t, \mathbf{b}^{(j)}\right)$ in (9). The corresponding expected length is $E\left[\tau_{j} \mid \mathbf{b}^{(j)}\right]=\int_{0}^{T}\left[1-F\left(t, \mathbf{b}^{(j)}\right)\right] d t$, and so using (9) and integrating we find

$$
\sum_{s \in \mathcal{S}} E\left[B_{s}^{(j)}\right] \beta_{s}^{(j)}=T_{j} \sum_{n=0}^{\infty} e^{-\Lambda, T_{j}} \frac{\left(\Lambda_{j} T_{j}\right)^{n}}{n !}\left\{\frac{\sum_{m=0}^{n}\left\|\boldsymbol{\theta}\left(m, \mathbf{b}^{(j)}\right)\right\|}{n+1}\right\} .
$$

Here $\pi\left(m, \mathbf{b}^{(j)}\right)=\pi\left(m-1, \mathbf{b}^{(j)}\right) \mathbf{W}^{(j)}$, where $\mathbf{W}^{(j)}$ is the transition matrix of the uniformized chain corresponding to $\mathcal{W}^{(j)}$, and $\pi\left(0, \mathbf{b}^{(j)}\right)=\mathbf{b}^{(j)}$.

We now find an expression for $E\left[V_{s}^{(j)}\right]$, which, in the case considered here, is the expected amount of time during a $j$-switchover interval when the particular queue $i$ has a specified number $q^{*}$ of customers. This length of time only depends on the size of queue $i$, say $q$, at the beginning of the switchover interval and the number of arrivals that occur to queue $i$, i.e., the number of arrivals from a Poisson process with rate $\lambda_{i}$ during a constant time period $\sigma_{j}$. We may assume that $q \leq q^{*}$, since the number of customers at any queue can only increase during a switchover interval (there are no departures from the polling system while the server is switching between queues). Thus it follows that $E\left[V_{s}^{(j)}\right]$ is the same for all initial states $s$ with the same $i$ th entry, i.e., for all $s \in \mathcal{S}_{q}^{(i)}$.

Condition on $n$, the number of arrivals to queue $i$ during a $j$-switchover interval ( $i$ and $j$ may or may not be equal). These $n$ arrivals split the switchover interval into $n+1$ subintervals, and since the arrival process to queue $i$ is Poisson, the subinterval lengths are exchangeable random variables $[3,4]$. It follows that the expected length of each subinterval is $\sigma_{j} /(n+1)$. Also, since there are no departures during a switchover interval, at most one of the subintervals can correspond to $q^{*}$ customers at queue $i$. In fact, there must be at least $q^{*}-q$ arrivals for the number of customers to reach $q^{*}$ at some time during the switchover period. Thus $E\left[V_{s}^{(j)}\right]=0$ if $n<q^{*}-q$, and $E\left[V_{s}^{(j)}\right]=\sigma_{j} /(n+1)$ if $n \geq q^{*}-q$. This gives

$$
\sum_{s \in \mathcal{S}} E\left[V_{s}^{(j)}\right] \alpha_{s}^{(j)}=\sum_{q=0}^{q^{*}} \sum_{n=q^{*}-q}^{\infty} e^{-\lambda_{i} \sigma_{j}} \frac{\left(\lambda_{i} \sigma_{j}\right)^{n}}{n !}\left(\frac{\sigma_{j}}{n+1}\right) a_{q}^{(i, j)} .
$$

Here, we have defined the vector $\mathbf{a}^{(i, j)}=\left\langle a_{0}^{(i, j)}, a_{1}^{(i, j)}, \ldots\right\rangle$, where (for $q=0,1, \ldots$ )

$$
a_{q}^{(i, j)}=\sum_{s \in \mathcal{S}_{q}^{(i)}} \alpha_{s}^{(j)}
$$

Equation (23) can also be written in the form

$$
\sum_{s \in \mathcal{S}} E\left[V_{s}^{(j)}\right] \alpha_{s}^{(j)}=\frac{1}{\lambda_{i}} \sum_{q=0}^{q^{*}} E_{q^{*}-q, \lambda_{i}}\left(\sigma_{j}\right) a_{q}^{(i, j)},
$$

where $E_{k, \lambda}(t)=1-\sum_{n=0}^{k} e^{-\lambda t}(\lambda t)^{n} / n !$ is the $(k+1)$-stage Erlangian distribution. This is the same as the result for the exhaustive discipline, since it only involves switchover intervals. 
The final quantity we need to deternine in order to use (20) for the gated system is $E\left[U^{(j)}\right]$, the expected time during a $j$-mini-cycle when there are a specified number of customers at a particular queue $i$. We first consider the case $i=j$, so that the queue of interest is also the queue $j$ being served. In order to calculate $E\left[U_{s}^{(j)}\right]$ in this case, it is necessary to determine the proportion of time during the mini-cycle that the uniformized chain $\mathcal{W}^{(j)}$ spends in states corresponding to, say $q^{*}$, total customers (original customers plus new arrivals) at the served queue. Note that $q^{-}>0$. since the server leaves queue $j$ whenever all original customers in the gated system finish service, and so the total number of customers at queue $j$ could never be 0 during the mini-cycle. In fact, only states of $\mathcal{W}^{(j)}$ of the form $(u, v)$ for which $v>0$ can contribute to the expected time $E\left[U_{s}^{(j)}\right]$, since the absorbing states $(u, 0)$ simply represent cases for which the server has already left queue $j$. It is for this reason that we can consider the behavior of the chain $W^{(j)}$ over the entire interval $\left(0, T_{j}\right)$ in our calculations, even if the timeout does not expire and $\tau_{j}<T_{j}$.

Since $E\left[U_{s}^{(j)}\right]$ only depends on the number of customers at queue $j$ at the beginning of the mini-cycle and not on the initial values for the other queues, this expectation has the same value for all $s \in \mathcal{S}_{q}^{(j)}$. Thus we can calculate $E\left[U_{*}^{(j)}\right]$ by using the initial stationary distribution $\mathbf{b}^{(j)}$ as was done for $E\left[B_{s}^{(j)}\right]$. In general, to find the expected amount of time that the served queue contains $q^{*}>0$ customers during the mini-cycle given an initial state distribution $\nu$ for the uniformized Markov chain $\mathcal{W}^{(j)}$, all states of the form $\left(q^{*}, v\right), v=1, \ldots, q^{*}$, must be included. The probability distribution for the uniformized chain at a time $t, 0 \leq t \leq T_{j}$, is given by $\mathbf{p}^{(j)}(t, \nu)$ in equation (8). Then the expected time in a particular set of states can be obtained by integrating (8) over the interval $\left(0, T_{j}\right)$. Using $\nu=b^{(j)}$ and performing the specified integration gives

$$
\sum_{s \in \mathcal{S}} E\left[U_{s}^{(j)}\right] \beta_{s}^{(i)}=T_{j} \sum_{n=0}^{\infty} e^{-\Lambda_{j} T_{j}} \frac{\left(\Lambda_{j} T_{j}\right)^{n}}{n !}\left\{\frac{\sum_{m=0}^{n} \sum_{v=1}^{q^{*}} \pi_{\left(q^{*}, v\right)}\left(m, \mathbf{b}^{(j)}\right)}{n+1}\right\},
$$

where $\pi\left(m, \mathbf{b}^{(j)}\right)=\pi\left(m-1, \mathbf{b}^{(j)}\right) \mathbf{W}^{(j)}$ and $\pi_{(q, q)}\left(0, \mathbf{b}^{(j)}\right)=\mathbf{b}_{q}^{(j)}\left(\right.$ so $\left.\pi_{(u, v)}\left(0, \mathbf{b}^{(j)}\right)=0, u \neq v\right)$.

When $j \neq i$, we wish to find the length of time during a $j$-mini-cycle that the nonserved queue $i$ has a total number, say $q^{*}$, of customers. This will depend, not only on the number $q$ initially at queue $i$. but also on the number $q^{\prime}$ initially at queue $j$, because the latter quantity determines the length $\tau_{j}$ of the mini-cycle. Thus, $E\left[U_{s}^{(j)}\right]$ is the same for all states that have identical $i$ th entries and identical $j$ th entries. Let us partition the state space according to these conditions. That is. for $q=0,1, \ldots, q^{\prime}=0,1, \ldots$, define $\mathcal{S}_{q, q^{\prime}}^{(i, j)}=\mathcal{S}_{q}^{(i)} \cap \mathcal{S}_{q^{\prime}}^{(j)}$. Also define the vectors $\mathbf{b}_{q}^{(i, j)}=\left\langle b_{q, 0}^{(i, j)}, b_{q, 1}^{(i, j)}, \ldots\right\rangle$, where

$$
b_{q, q^{\prime}}^{(i, j)}=\sum_{s \in \mathcal{S}_{q, q^{\prime}}^{(i, j)}} \beta_{s}^{(j)}
$$

To determine the common value $E\left[U_{s}^{(j)}\right]$ for $s \in \mathcal{S}_{q, q^{\prime}}^{(i, j)}$, we need only consider the case $q^{*} \geq q$, since no departures can occur at queue $i$. Then using arguments about exchangeable random variables similar to those given above, we have using the law of total probability

$$
\begin{aligned}
\sum_{s \in \mathcal{S}} E\left[U_{s}^{(j)}\right] \beta_{s}^{(j)}= & \sum_{q=0}^{q^{*}} \sum_{n=q^{*}-q}^{\infty} e^{-\lambda_{i} T_{j}} \frac{\left(\lambda_{i} T_{j}\right)^{n}}{n !}\left(\frac{T_{j}}{n+1}\right) P\left[\tau_{j}=T_{j} \mid \mathbf{b}_{q}^{(i, j)}\right] \\
& +\sum_{q=0}^{q^{*}} \int_{0}^{T_{j}} \sum_{n=q^{*}-q}^{\infty} e^{-\lambda_{i} t} \frac{\left(\lambda_{i} t\right)^{n}}{n !}\left(\frac{t}{n+1}\right) F^{\prime}\left(t, \mathbf{b}_{q}^{(i, j)}\right) d t .
\end{aligned}
$$


The first sum, say $\mathcal{C}_{1}$, represents the contribution to the expectation from those cases for which the timeout expires $\left(\tau_{j}=T_{j}\right)$, while the second sum, say $\mathcal{C}_{2}$, covers the cases for which the server leaves before the timeout expires $\left(\tau_{j}<T_{j}\right)$. Evaluating these quantities, we see that the first sum is

$$
\begin{aligned}
\mathcal{C}_{1}= & \sum_{q=0}^{q^{*}} \sum_{n=q^{*}-q}^{\infty} \sum_{k=n}^{\infty} \epsilon^{-\left(\lambda_{1}+\Lambda_{\jmath}\right) T_{j}} \frac{\left[\left(\lambda_{i}+\Lambda_{j}\right) T_{j}\right]^{k}}{k !} \\
& \times\left(\frac{\lambda_{i}}{\lambda_{i}+\Lambda_{j}}\right)^{n}\left(\frac{\Lambda_{j}}{\lambda_{i}+\Lambda_{j}}\right)^{k-n}\left(\begin{array}{l}
k \\
n
\end{array}\right)\left(\frac{T_{j}}{n+1}\right)\left\|\theta\left(k-n, \mathbf{b}_{q}^{(i, j)}\right)\right\| .
\end{aligned}
$$

Here $\pi\left(m, \mathbf{b}_{q}^{(i, j)}\right)=\pi\left(m-1, \mathbf{b}_{q}^{(i, j)}\right) \mathbf{W}^{(j)}$ and $\pi\left(0, \mathbf{b}_{q}^{(i, j)}\right)=\mathbf{b}_{q}^{(i, j)}$. In equation (26), $q$ represents the initial state of queue $i, n$ represents the number of arrivals to queue $i$, and $k$ represents the number of events of the superposition of two processes, namely, the Poisson arrival process to queue $i$ with rate $\lambda_{i}$, and the uniformized Markov chain for queue $j$ with rate $\Lambda_{j}=\lambda_{j}+\mu_{j}$. The second sum is

$$
\begin{aligned}
\mathcal{C}_{2}= & \sum_{q=0}^{q^{*}} \sum_{n=q^{*}-q}^{\infty} \sum_{k=n+1}^{\infty} e^{-\left(\lambda_{i}+\Lambda_{j}\right) r_{j}} \frac{\left[\left(\lambda_{i}+\Lambda_{j}\right) T_{j}\right]^{k}}{k !} \\
& \times \sum_{m=n+1}^{k}\left(\frac{\lambda_{i}}{\lambda_{i}+\Lambda_{j}}\right)^{n}\left(\frac{\Lambda_{j}}{\lambda_{i}+\Lambda_{j}}\right)^{m-n}\left(\begin{array}{c}
m-1 \\
n
\end{array}\right)\left[\frac{m T_{j} /(k+1)}{n+1}\right]\left\|\phi\left(m-n, \mathbf{b}_{q}^{(i, j)}\right)\right\| .
\end{aligned}
$$

In equation (27), $q$ represents the initial state of queue $i, n$ represents the number of arrivals to queue $i, k$ represents the number of events of the superposition of the arrival process to queue $i$ and the uniformized Markov chain for queue $j$, and $m$ represents the event at which the server departs from queue $j$ (i.e., the uniformized Markov chain $\mathcal{W}^{(j)}$ reaches an absorbing state). Also, $m T_{j} /(k+1)$ corresponds to the expected length of time until the server leaves queue $j$ (i.e., that portion of the uniformization interval $\left(0, T_{j}\right)$ during which arrivals to queue $i$ may occur).

\section{EXAMPLES}

In this section we present simple examples to illustrate the applicability of the method we developed in previous sections. In the first example we compare the gated and exhaustive policies. Consider a multiplexor for voice and data packets which uses a time limit service policy for each type of stream as shown in Figure 1. The voice and data streams of packets are served one after the other, and both streams use the same discipline, either exhaustive or gated. When the server starts serving the voice packets, a timeout of length $T_{1}$ is activated. If the timeout expires, the server immediately switches to the data queue. The behavior at the data queue is similar, except that the timeout is of length $T_{2}$.

For the first example, we assume that, for both queues, the channel capacity is $1.5 \mathrm{Mbps}$, the voice load is $60 \%$ of the capacity, the data load varies from $10 \%$ to $60 \%$ of the total capacity, and the buffer size is equal to 10 packets. The average size of the data and voice packets is 400 and $600 \mathrm{bits}$, respectively, and the switchover time is $0.1 \mathrm{msec}$. In Figure 2 we compare the average packet delay for voice and data packets when the service policy at both queues is exhaustive and when the policy at both queues is gated. In that figure two sets of timeout values are used: $T_{1}=8 \mathrm{msec}, T_{2}=1 \mathrm{msec}$, and $T_{1}=8 \mathrm{msec}, T_{2}=2 \mathrm{msec}$. We note in both cases that voice 


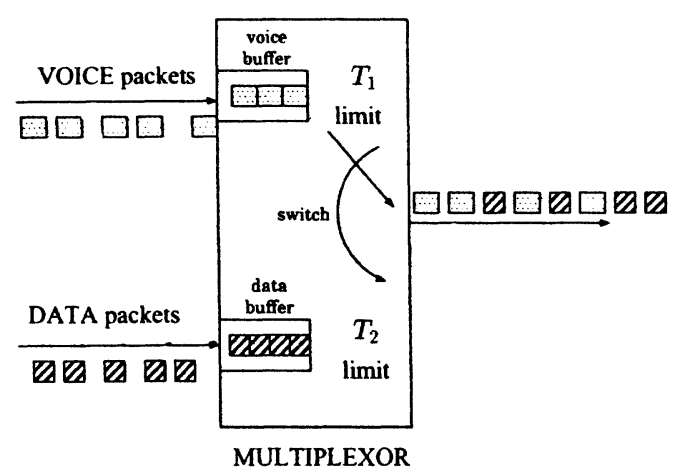

Figure 1: Multiplexor for voice and data.

delay, when the gated discipline is used, is always greater than the corresponding delay under the exhaustive discipline. This increase in the voice delay is compensated for by the decrease in the data delay. Note also that the percentage increase in the voice delay is much larger than the decrease in the data delay, especially for high values of the data load. Even when the timeout values for data packets are kept small $\left(T_{2}=1\right)$ and a minimum of $T_{1} /\left(T_{1}+T_{2}\right)=89 \%$ of the capacity is guaranteed for the voice traffic under both the exhaustive and the gated disciplines, the "protection" that the timeouts provide for voice packets is smaller under the gated policy and the delay is more sensitive to an increase in the data load than under the exhaustive policy. This may be explained by the high value of $T_{1}$ when compared to the expected service times of voice packets. In the gated system, the multiplexor may switch to the data queue even if the timeout has not yet expired and there are voice packets to be served. But in the exhaustive system, if the timeout has not expired, then the server keeps serving the voice packets until the queue empties. This observation is strongly dependent on the timeout values used.

In Figure 3 we compare the blocking probabilities for voice packets for the exhaustive and gated systems, with the same parameter values as before and when the buffer size increases by $50 \%$. As expected from the previous set of figures, the blocking probabilities for voice packets when the gated discipline is used is greater than the corresponding probabilities under the exhaustive discipline. The difference between the two disciplines is much higher for large values than for small values of data load.

The second example compares the expected delay and blocking probabilities for a gated system having preemptive timeouts with one having nonpreemptive timeouts, and the results are shown in Figure 4. The parameter values are the same as the first example. In Figure 4 note that the system provides less "protection" to the voice packets when overruns are allowed, and the average delay of voice increases (by at most $5 \%$ in this example). On the other hand, the average delay of data packets decreases (by less than $5 \%$ at most). Note that this behavior cannot be immediately concluded based on the parameter values used. From the same figure we also note that, as expected from the delay curves, the blocking probabilities for the voice packets increase (by about $10 \%$ at most), while the blocking probabilities for data packets decrease. 


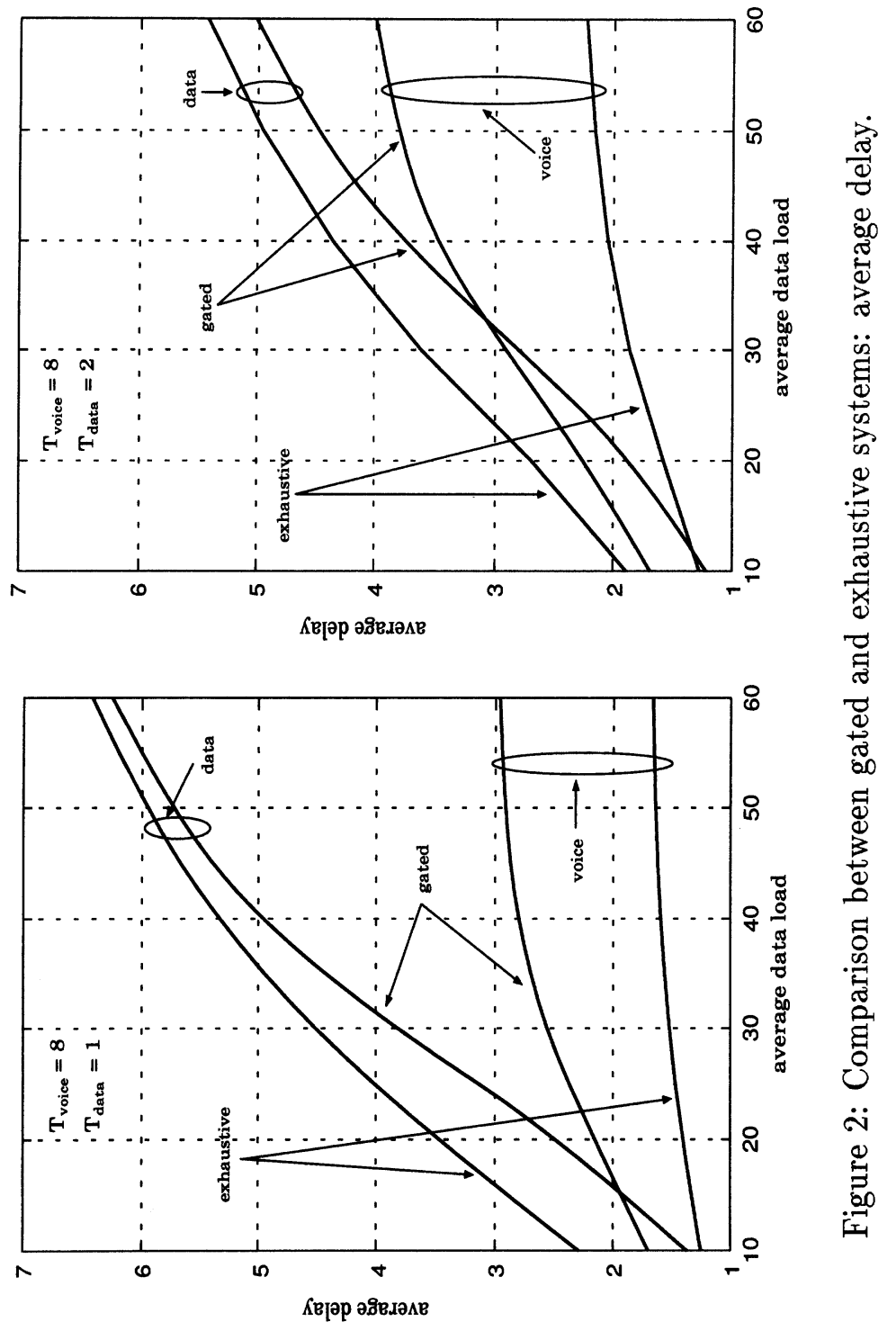




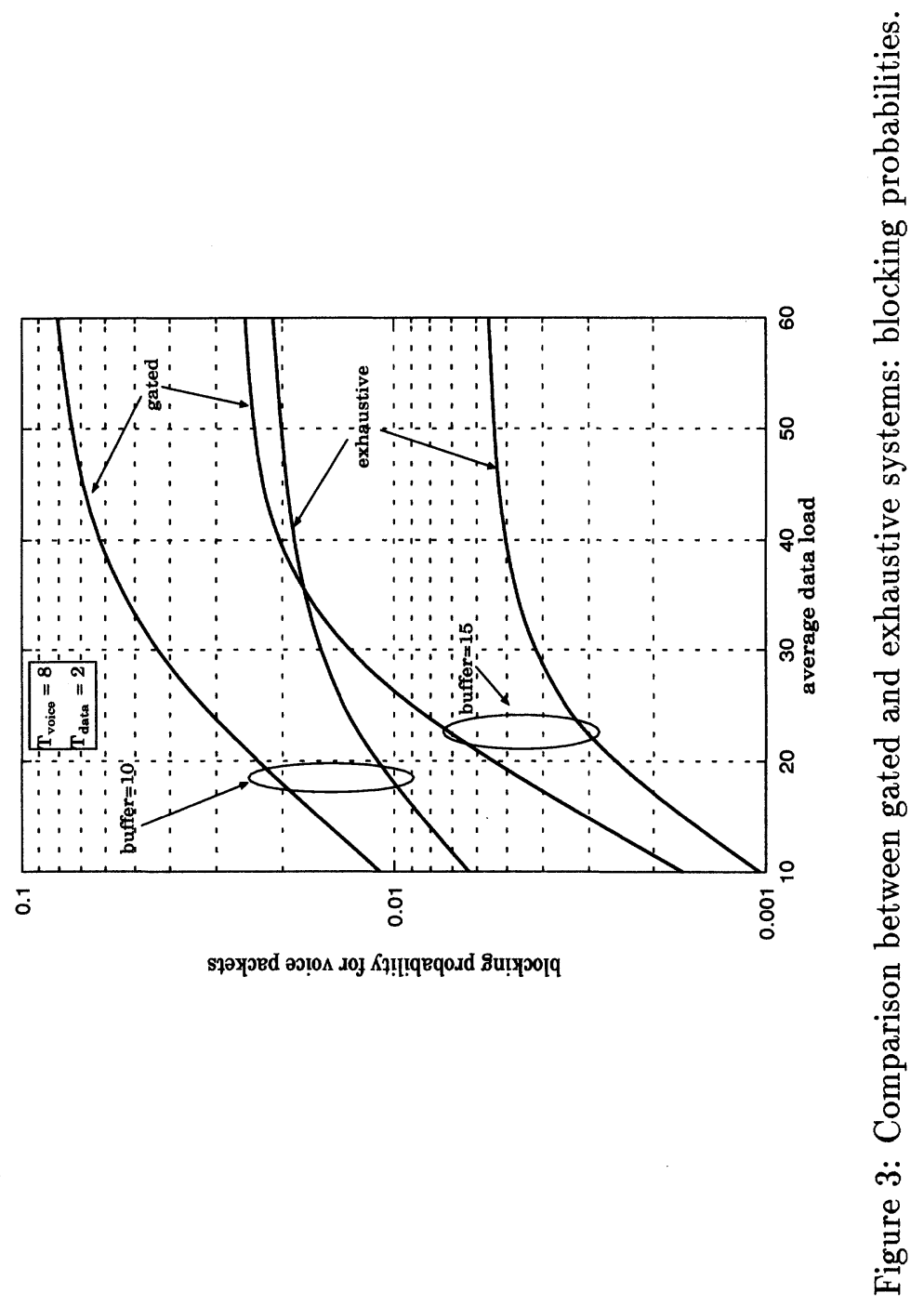




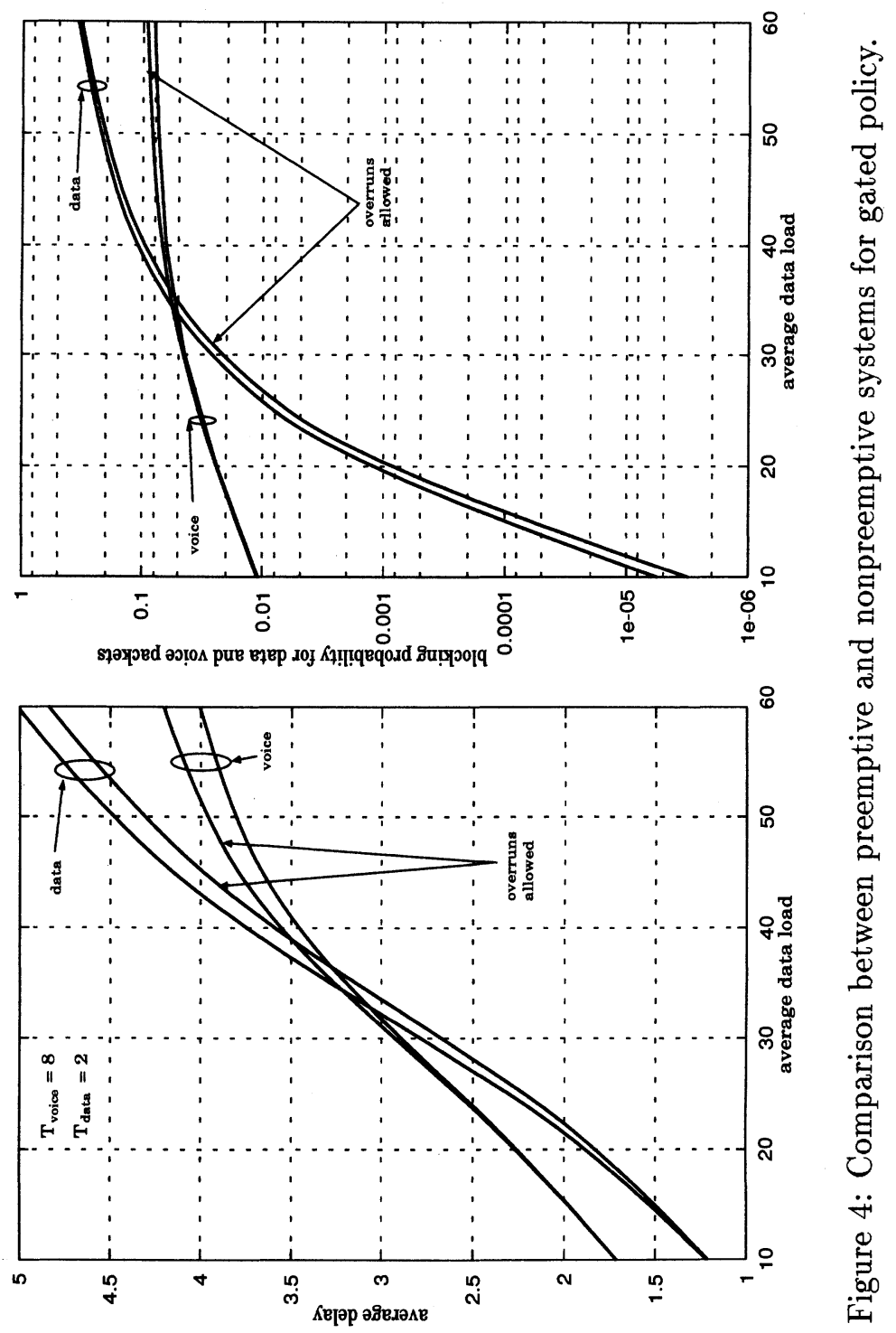




\section{Acknowledgment}

We wish to thank Morganna Carmen Diniz for implementing the algorithms presented here.

\section{REFERENCES}

[1] D. Bertsekas and R. Gallager. Data Networks, 2nd Ed. Prentice-Hall, 1992.

[2] E.G. Coffman, Jr., G. Fayolle, and I. Mitrani. Two queues with alternating service periods. In Proc. Performance '87, pages 227-239, 1987.

[3] H.A. David. Order Statistics, 2nd Ed. John Wiley \& Sons, 1981.

[4] E. de Souza e Silva and H.R. Gail. Calculating availability and performability measures of repairable computer systems using randomization. Journal of the ACM, 36(1):171-193, 1989.

[5] E. de Souza e Silva and H.R. Gail. Performability analysis of computer systems: from model specification to solution. Performance Evaluation, 14:157-196, 1992.

[6] E. de Souza e Silva and H.R. Gail. The uniformization method in performability analysis. In Proceedings of the 2nd International Workshop on Performability Analysis, 1993.

[7] E. de Souza e Silva, H.R. Gail, and R.R. Muntz. Polling systems with server timeouts and their application to token passing networks. IEEE/ACM Trans. on Networking, 3(5):560$575,1995$.

[8] B.L. Fox and P.W. Glynn. Computing Poisson probabilities. Communications of the ACM, $31(4): 440-445,1988$.

[9] W.K. Grassmann. Méans and variances of time averages in Markovian environments. European Journal of Operational Research, 31:132-139, 1987.

[10] W.K. Grassmann. Finding transient solutions in Markovian event systems through randomization. In Numerical Solution of Markov Chains, pages 357-3i1. Marcel Dekker, Inc., 1991.

[11] D. Gross and D.R. Miller. The randomization technique as a modeling tool and solution procedure for transient Markov processes. Operations Research, 32(2):343-361, 1984.

[12] K.K. Leung and M. Eisenberg. A single-server queue with vacations and gated time-limited service. IEEE Trans. on Communications, 38(9):1454-1462, 1990.

[13] K.K. Leung and M. Eisenberg. A single-server queue with vacations and non-gated timelimited service. Performance Evaluation, 12(2):115-125, 1991.

[14] K. Sriram. Dynamic bandwidth allocation and congestion control schemes for voice and data multiplexing in wideband and packet technology. In ICC-90, pages 1003-1009, 1990.

[15] H. Takagi. Analysis of Polling Systems. MIT Press, 1986. 
[16] H. Takagi. Queueing analysis of polling models. ACM Computing Surveys, 20(1):5-28, 1988.

[17] H. Takagi. Queueing analysis of polling models: an update. In Stochastic Analysis of Computer and Communication Systems, pages 267-318. North-Holland, 1990.

[18] O.-C. Yue and C.A. Brooks. Performance of the timed token scheme in MAP. IEEE Trans. on Communications, 38(7):1006-1012, 1990. 